\title{
miR-455 Inhibits the Viability and Invasion by Targeting RAB18 in Hepatocellular Carcinoma
}

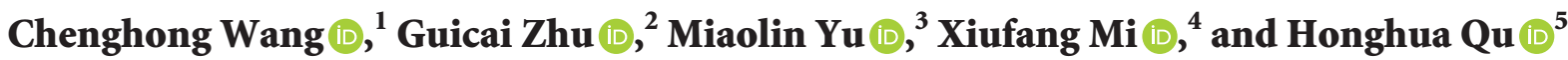 \\ ${ }^{1}$ Department of Clinical Laboratory, Yantaishan Hospital, Yantai 264000, China \\ ${ }^{2}$ Department of Surgery, Rizhao Hospital of TCM, Rizhao 276800, China \\ ${ }^{3}$ Department of Chinese Medicine, Qingdao Central Hospital, Qingdao University, Qingdao 266042, China \\ ${ }^{4}$ Department of Internal Medicine, Zhangqiu District People's Hospital, Jinan 250200, China \\ ${ }^{5}$ Department of Medical Insurance Office, Qilu Hospital of Shandong University, Jinan 250012, China \\ Correspondence should be addressed to Honghua Qu; quhonghua@sdqiluhospital.cn
}

Received 29 March 2021; Accepted 19 May 2021; Published 28 May 2021

Academic Editor: Alamgeer Yuchi

Copyright (c) 2021 Chenghong Wang et al. This is an open access article distributed under the Creative Commons Attribution License, which permits unrestricted use, distribution, and reproduction in any medium, provided the original work is properly cited.

\begin{abstract}
Background. Hepatocellular carcinoma (HCC) has been regarded as the fifth most common cancer worldwide with a low prognosis. miR-455 usually played the role of a tumor suppressor in multiple cancers. The aim of this study was to investigate the roles of miR-455 in HCC. Materials and Methods. Cell viability and invasion were measured by CCK8 and Transwell assays. Luciferase reporter assay was performed to verify that miR- 455 directly binds to the $3^{\prime}$-noncoding region (UTR) of RAB18 mRNA in Huh7 cells. Results. The expression of miR-455 was lower in HCC tissues and cell lines than in nontumor tissues and normal cell line, and downregulation of miR-455 was connected with worse outcome of HCC patients. miR-455 suppressed cell proliferation in vitro and in vivo, and it inhibited the abilities of cell invasion and EMT in HCC. RAB18 was upregulated in HCC tissues and cell lines, and the expression of RAB18 was regulated by miR-455. RAB18 reversed partial roles of miR-455 on cell viability and invasion in HCC. Conclusion. miR-455 inhibited cell viability and invasion by directly targeting the $3^{\prime}$-UTR of RAB18 mRNA of hepatocellular carcinoma.
\end{abstract}

\section{Introduction}

Hepatocellular carcinoma (HCC), the third major cause of cancer-related death, is the fifth most common cancer worldwide [1]. In recent years, the increase in the incidence of HCC has been the result of a combination of factors, especially the phenotype caused by hepatitis $\mathrm{B}$ or $\mathrm{C}$ virus (HBV or HCV) infection [2, 3]. Despite significant advances in treatment and diagnosis, surgery is the primary treatment for patients with HCC. About half of HCC cases are advanced unresectable HCC, resulting in a poor prognosis [4]. Thus, it is necessary to investigate the biomarkers for the treatment and the pathogenesis of HCC.

MicroRNAs (miRNAs) are small noncoding endogenous RNAs containing 19 to 25 nucleotides that promote posttranscriptional control in regulating the expression of target gene by binding to the $3^{\prime}$-UTR sequences of its mRNA [5]. Recently, increasing evidences elucidated that miRNAs was involved in the cancer pathogenesis, including cell proliferation, metastasis, and apoptosis [6]. Most reports found that multiple miRNAs that include miR-548a, miR-1246, miR-632, and miR-5692a played pivotal roles in HCC [7-10]. miR-455 has been reported to act as a tumor suppressor to inhibit cap-dependent translation and the proliferation in prostate cancer [11]. Also, in gastric cancer, miR-455 inhibited human cell proliferation and invasion and promoted cell apoptosis [12]. In addition, miR-455 inhibited cell viability, while it induced cell apoptosis in colorectal cancer [13]. However, miR-455 promoted cell invasion and migration in triple-negative breast cancer [14]. Thus, the pivotal roles of miR-455 in cell viability and metastasis in HCC still needed to be explored. 
RAB18, a member of Ras-related small GTPases family, belongs to members of the Ras oncogene superfamily of small guanosine triphosphatases [15]. Accumulating evidences have elucidated connection between the expression of GTPases members and several diseases, including colorectal cancer, lung cancer, and prostate cancer [16-18]. RAB18 regulates membrane trafficking in organelles and transport vesicles, leading to a reduction in mature LDs and lipid storage [19]. RAB18 binds to NS5A to improve the interaction between sites of viral replication and lipid droplets [20]. RAB18 is reduced in pituitary tumors, resulting in acromegaly and restoration of excessive growth hormone hypersecretion [21]. RAB18 was associated with lipogenesis, lipolysis, and obesity in adipocytes [22]. However, the functions of RAB18 in HCC remain unclear; thus, in the present study, we discovered that miR-455 inhibited cell viability, invasion, and EMT by directly targeting to the $3^{\prime}$-UTR of RAB18 mRNA in hepatocellular carcinoma.

\section{Materials and Methods}

2.1. Patients and Tissue Samples. A cohort of 98 patients who underwent HCC were harvest from Yantaishan Hospital during the period from January 2016 to November 2018. None of the patients received preoperative treatment such as chemotherapy and radiotherapy before surgery. All the fresh tissues were snap-frozen in liquid nitrogen and stored at $-80^{\circ} \mathrm{C}$ until RNA extraction. The project protocol was reviewed and approved by the Ethics Committee of Zhangjiagang Hospital. All participants signed the written informed consents before inclusion in this study.

2.2. Cell Culture. HCC cell lines HCC-LM3, Huh7, and Bel7402 and a normal liver cell L-O2 were obtained from American Type Culture Collection (ATCC, Rockville, MD, USA). All cells were cultured with Dulbecco's modified Eagle's medium (DMEM, Invitrogen, CA, USA) supplemented with $10 \%$ FBS (Gibco, USA) at $37^{\circ} \mathrm{C}$ in a humidified atmosphere containing $5 \% \mathrm{CO}_{2}$.

2.3. Western Blot. Radioimmunoprecipitation assay (RIPA, Thermo Fisher Scientific, Waltham, MA, USA) lysis buffer containing PMSF was employed to separate total protein on ice for $37^{\circ} \mathrm{C}$. After centrifugation at $12,000 \times \mathrm{g}$ for $20 \mathrm{~min}$ at $4^{\circ} \mathrm{C}$, the concentration of the protein was then quantified using BCA Protein Assay Reagent Kit (Beyotime, Shanghai, China). Equal amounts of protein for each sample were loaded and separated on a $10 \%$ sodium dodecyl sulphatepolyacrylamide gel (SDS-PAGE) before being transferred onto a polyvinylidene difluoride (PVDF, Roche Diagnostics) membrane. After blocking in 5\% skim milk powder at room temperature for $1 \mathrm{~h}$, the membrane was incubated by primary antibodies overnight at $4^{\circ} \mathrm{C}$. The primary antibodies were RAB18 (1:1000, Santa Cruz Biotechnology, Santa Cruz, CA, USA), E-cadherin, N-cadherin, and glyceraldehyde-3phosphate dehydrogenase (GAPDH). Next, horseradish peroxidase- (HRP-) conjugated secondary antibody (1:5000) was conducted to incubate the membrane at room temperature for $1 \mathrm{~h}$. The Enhanced Chemiluminescence (ECL) Kit (KeyGen Biotech, China) was applied to measure the bands and imaged on a Tanon-5200 Chemiluminescent Imaging System (Millipore, Bedford, MA, USA).

2.4. RNA Isolation and $q R T-P C R$. TRIzol reagent (Invitrogen, USA) was used to extract the total RNAs from tissues and cell lines. The miRNA was reverse-transcribed using miRNA first-strand complementary DNA (cDNA) synthesis kit (Poly A Tailing; Sangon, China), while mRNA was reverse-transcribed using RevertAid First-Strand cDNA Synthesis Kit (Thermo Fisher Scientific). Subsequently, qRTPCR was carried out using SYBR Premix Ex Taq kit (Takara) on an ABI PRISM 7900 Sequence Detection System (Applied Biosystems). The $2^{-\Delta \Delta \mathrm{Cq}}$ method was applied to analyze the miRNA or mRNA expression with GAPDH or U6 was the normalization. The primer pairs are shown in Table 1.

2.5. Cell Proliferation Assay. The cell proliferation was calculated using cell counting kit-8 (CCK-8) assay (Dojindo Laboratories, Kumamoto, Japan) in 96-well plates. After 24, 48,72 , or $96 \mathrm{~h}$ of culture, CCK-8 solution was added to each well and cultivated for $3 \mathrm{~h}$ at $37^{\circ} \mathrm{C}$. The plates were shook for $20 \mathrm{~min}$, followed by measuring the absorbance at $450 \mathrm{~nm}$ using automatic multiwell spectrophotometer (Bio-Rad, Richmond, CA, USA).

2.6. Invasion Assay. Invasive ability was evaluated using Transwell insert (Corning Incorporated, Corning, New York) covered with Matrigel (BD Biosciences). Briefly, the cells were suspended in DMEM medium without FBS and the cell suspension was placed in the upper well, while the bottom well was filled with medium with $20 \%$ FBS that acted as chemoattractant. After incubation at $37^{\circ} \mathrm{C}$ with $5 \% \mathrm{CO}_{2}$ for $24 \mathrm{~h}$, the cells that failed to pass through the membrane were removed with cotton swabs. Meanwhile, the cells that passed through the membrane were fixed with $100 \%$ methanol for $15 \mathrm{~min}$ and stained using $0.1 \%$ crystal violet solution for $30 \mathrm{~min}$. Finally, a microscope (Olympus, Tokyo, Japan) was utilized to count the invaded cells.

2.7. Cell Transfection. The miR-455 mimic, miR-455 inhibitor, and RAB18 overexpression plasmids, as well as corresponding negative control, were obtained from GenePharma (Shanghai, China). Cells were placed in a 6-well plate, and the transfections of special vectors were performed using Lipofectamine 2000 Reagent (Invitrogen, Carlsbad, CA, USA) following the manufacturer's instruction.

2.8. Dual-Luciferase Reporter Gene Assay. TargetScan predicted the miR-455 binding site at $3^{\prime}$-UTR of RAB1 8 mRNA. The putative sequences of miR- 455 on RAB18 mRNA were mutated from GCACAUA to CGUGUAU. The wild type or the mutated sequences of RAB18 mRNA were cloned into pmiRGLO Vector, which were designated as pmiRGLO- 
Table 1: Primer sequences for RT-qPCR.

\begin{tabular}{|c|c|c|}
\hline Gene & & Primer sequences \\
\hline$m i R-455$ & $\begin{array}{l}\text { Forward } \\
\text { Reverse }\end{array}$ & $\begin{array}{c}5^{\prime} \text {-CGAGCTTCCTTCTGCAGGT-3' } \\
5^{\prime} \text {-CACCACTGCCATCCCACA-3 }\end{array}$ \\
\hline U6 & & $\begin{array}{c}5^{\prime} \text {-TGCGGGTGCTCGCTTCGCAGC-3' } \\
5^{\prime} \text {-CCAGTGCAGGGTCCGAGGT-3' }\end{array}$ \\
\hline$R A B 18$ & & $\begin{array}{c}5^{\prime} \text {-CAGGGAAGAAGGCCAAGGAG-3' } \\
5^{\prime} \text {-CCCGGGGTCGATGGAGT-3' }\end{array}$ \\
\hline GAPDH & $\begin{array}{l}\text { Forward } \\
\text { Reverse }\end{array}$ & 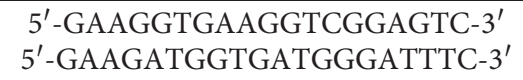 \\
\hline
\end{tabular}

RAB18-WT (WT) and pmiRGLO-RAB18-MUT (MUT), respectively. The Huh7 cells were seeded and cotransfected with pmiRGLO-RAB18-WT or pmiRGLO-RAB18-MUT plasmid and miR-455 mimic or miR-455 NC using Lipofectamine 2000 (Invitrogen, Carlsbad, CA, USA). Firefly luciferase activity was assessed by dual-luciferase reporter assay system (Promega, Madison, WI, USA), with Renilla luciferase activity acting as normalization.

2.9. Construction of the Mice Xenograft Model. Four-week-old nude mice were purchased from Vital River Laboratory Animal Technology (Beijing, China). Huh7 cells that transfected miR-455 mimic or control plasmids were subcutaneously inoculated in the mice to build xenograft tumor model. The volumes were calculated as $1 / 2 \times$ length $\times$ width $^{2}$ of the tumor, which were measured every 3 days. After cultivation for 26 days, the mice were executed and the tumors dissected out. The animal study was also approved by the Research Ethics Committee of Zhangjiagang Hospital.

2.10. Statistical Analysis. The data were indicated as means \pm SD. The SPSS statistical software version 19.0 (IBM Corp, Armonk, NY, USA) and GraphPad Prism 7.0 (GraphPad, San Diego, CA) were used to analyze all the data. The differences between two or more groups were compared by Student's $t$-test or one-way analysis of variance (ANOVA) followed by the Student-Newman-Keuls post hoc test. Values of $P<0.05$ were considered to be statistically significant.

\section{Results}

3.1. The Expression of miR-455 in HCC. To investigate the functions of miR-455 in HCC, qRT-PCR was conducted to measure the expression of miR-455 in tissues or cell lines. As a result, miR-455 had a low expression in HCC tissues versus noncancerous tissues $(P<0.05)$ (Figure $1(\mathrm{a}))$. The expression of miR-455 was also evaluated in HCC cell lines HCCLM3, Huh7, and Bel-7402 and normal liver cell L-O2. As expected, the expression of miR-455 was lower in HCC cell lines HCC-LM3 $(P<0.05)$, Huh7 $(P<0.01)$, and Bel-7402 $(P<0.05)$ than in L-O2 cells (Figure $1(b))$.

3.2. miR-455 Inhibits Cell Viability of HCC In Vitro and In Vivo. To explore the roles of miR-455 in cell proliferation,
miR-455 mimic and miR-455 inhibitor were utilized to upregulate $(P<0.01)$ or downregulate $(P<0.05)$ the expression of miR-455 in HCC cells Huh7, which was measured by RT-qPCR (Figure 2(a)). CCK8 assay showed that the proliferative ability was decreased by transfecting miR-455 mimic $(P<0.05)$ (Figure $2(b))$. On the contrary, miR-455 inhibitor increased the proliferative ability in comparison with normal control $(P<0.05)$ (Figure 2(c)).

In addition, Huh7 cells stably transfected with miR-455 mimic had a slower growth rate in vivo (Figure 2(d)). After 26 days of cultivation, the nude mice were executed and the volume of the xenograft tumor was calculated. Not unfortunately, tumors of miR-455 overexpressed group had a smaller volume than that of control group $(P<0.05)$ (Figure 2(e)). All results elucidated that miR-455 suppressed the growth of HCC in vitro and in vivo.

3.3. miR-455 Impairs Cell Invasion and the EMT in HCC Cells. Transwell assay was performed to calculate cell invasion in Huh7 cells transfected with miR-455 mimic or miR-455 inhibitor. As expected, the invasive ability was reduced by miR-455 mimic $(P<0.05)$, while it was improved by miR455 inhibitor in Huh7 cells $(P<0.05)$ (Figure 3(a)). Furthermore, proteins associated with EMT markers, such as E-cadherin and $\mathrm{N}$-cadherin, were measured using western blot. As a result, the expression of $\mathrm{N}$-cadherin was inhibited, while the expression of E-cadherin was improved by miR455 mimic. On the contrary, miR-455 inhibitor enhanced the expression of $\mathrm{N}$-cadherin, whereas it decreased the expression of E-cadherin in Huh7 cells (Figure 3(b)).

3.4. miR-455 Directly Targets the 3'-UTR of RAB18 mRNA. TargetScan predicted RAB18 was a potential target of miR455 at $96-102$ on the $3^{\prime}$-UTR. To verify miR-455 binding to the $3^{\prime}$-UTR of RAB18 mRNA, the putative binding sequences were mutated from GCACAUA to CGUGUAU, as shown in Figure 4(a). The miR-455 mimic and the wild type or the mutant $3^{\prime}$-UTR were cotransfected in Huh7 cells, followed by calculating the luciferase ability. As expected, miR-455 mimic reduced the luciferase ability of wild type mRNA $3^{\prime}$-UTR $(P<0.05)$, whereas it did not alter the mutant $3^{\prime}$-UTR $(P>0.05)$ (Figure $\left.4(b)\right)$. Furthermore, the expression of RAB18 was assessed after transfection with miR-455 mimic or inhibitor. The expression of RAB18 was decreased by miR-455 mimic $(P<0.05)$, while it was enhanced by miR-455 inhibitor in Huh7 cells $(P<0.05)$ (Figure 4(c)).

3.5. RAB18 Restores Partial Functions of miR-455 on Cell Viability and Invasion. To investigate the roles of RAB18 in HCC, the expression of RAB18 was assessed in tissues and cell lines by RT-qPCR. Not unfortunately, RAB18 was overexpressed in HCC tissues versus nontumor tissues (Figure 5(a)). In cells, the expression of RAB18 was higher in HCC-LM3 $(P<0.05)$, Huh7 $(P<0.01)$, and Bel-7402 $(P<0.05)$ cells than in L-O2 cells (Figure 5(b)). To investigate the inhibitory effect of miR-455 on cell proliferation and invasion CCK8 and 


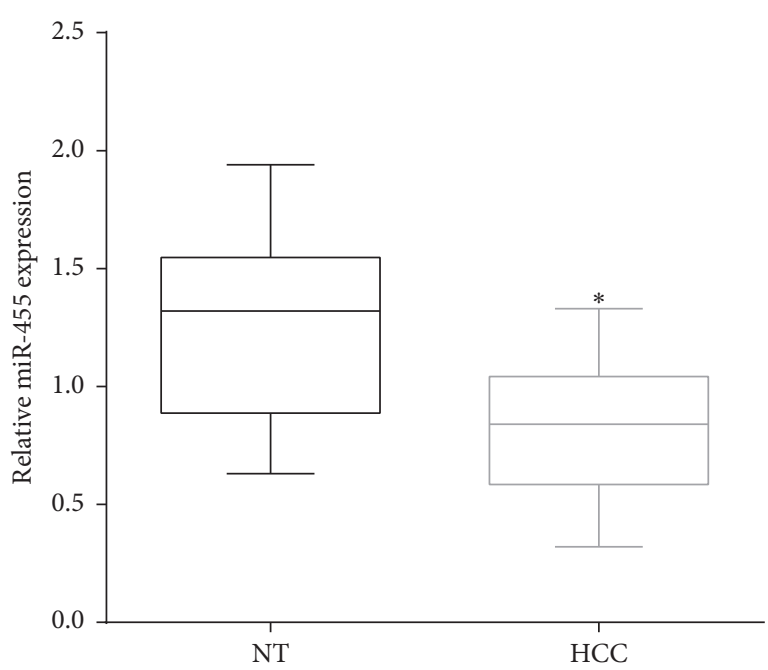

(a)

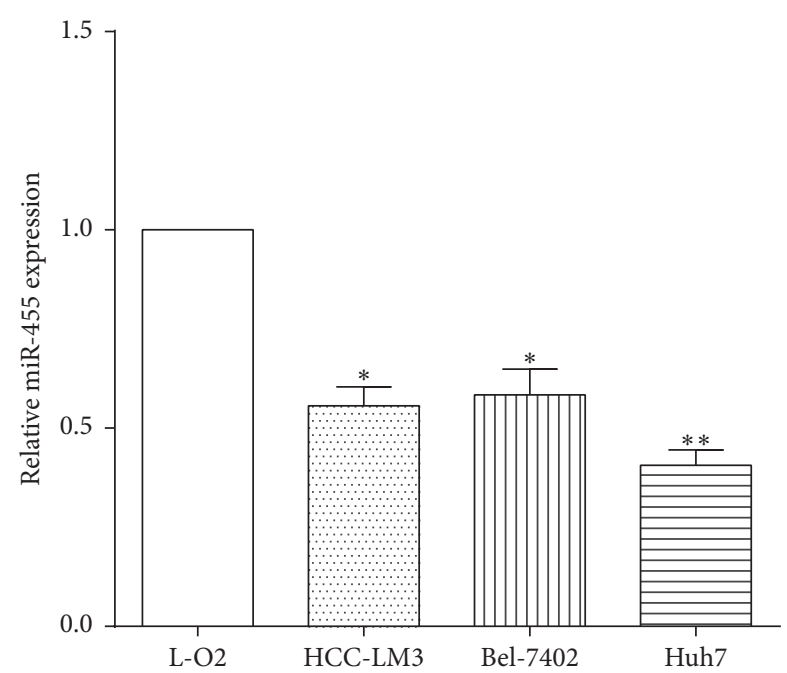

(b)

Figure 1: The expression of miR-455 in HCC. (a) The expression of miR-455 was low in HCC tissues versus noncancerous tissues vs NT, ${ }^{*} P<0.05$. (b) The expression of miR-455 was lower in HCC cells than in normal cells vs L-O2, ${ }^{*} P<0.05$; ${ }^{* *} P<0.01$.

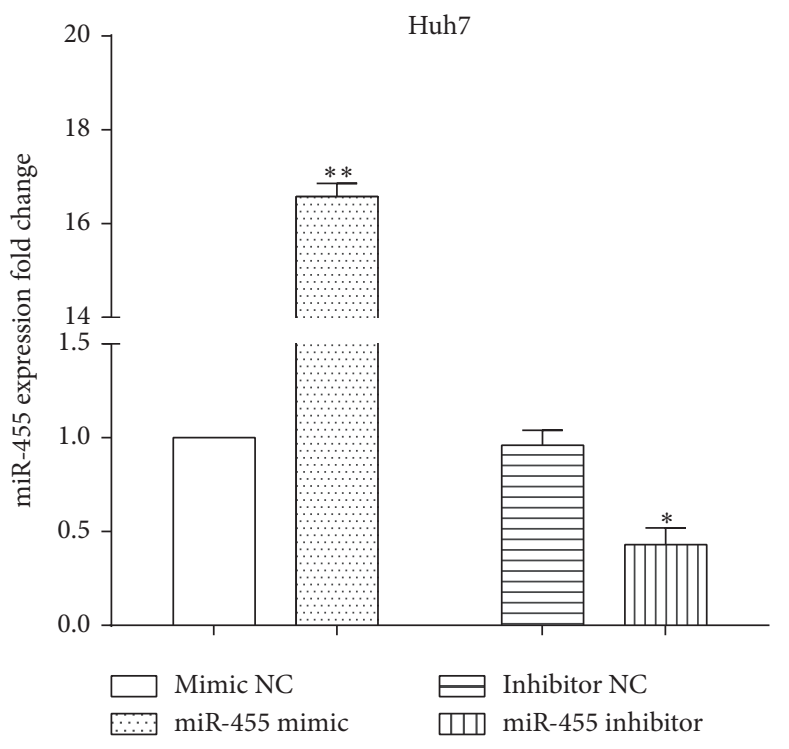

(a)

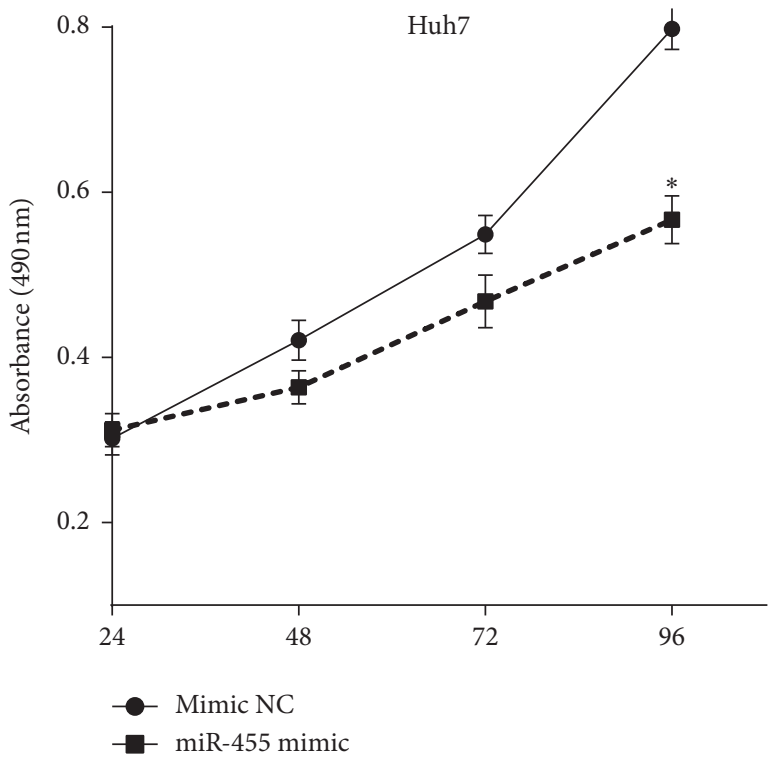

(b)

FIGURE 2: Continued. 


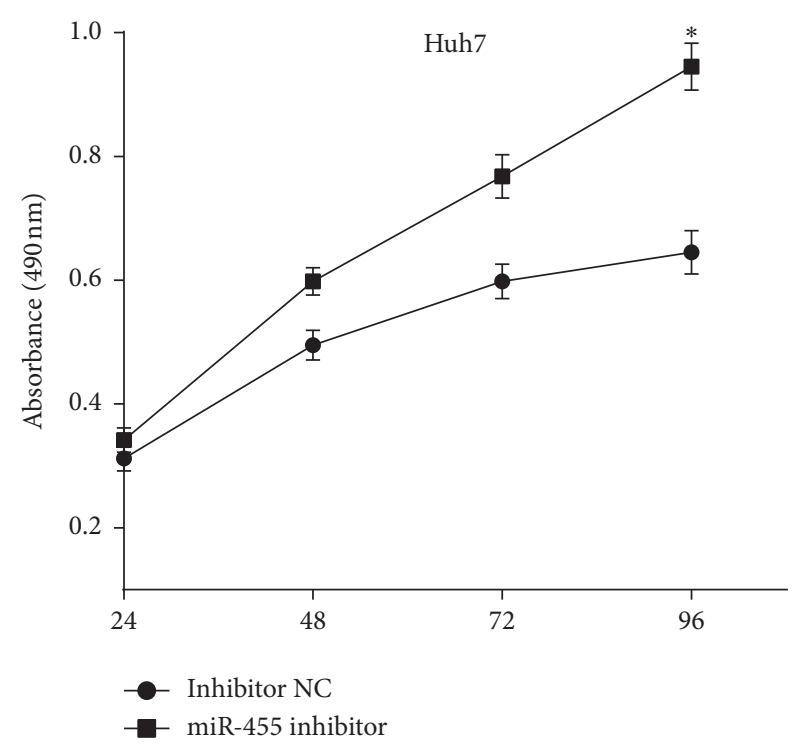

(c)

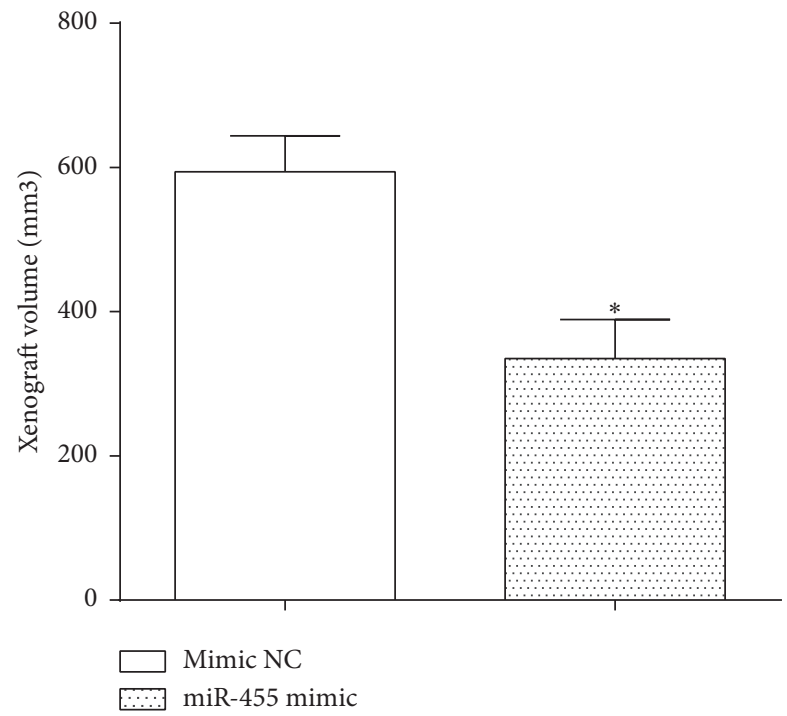

(e)

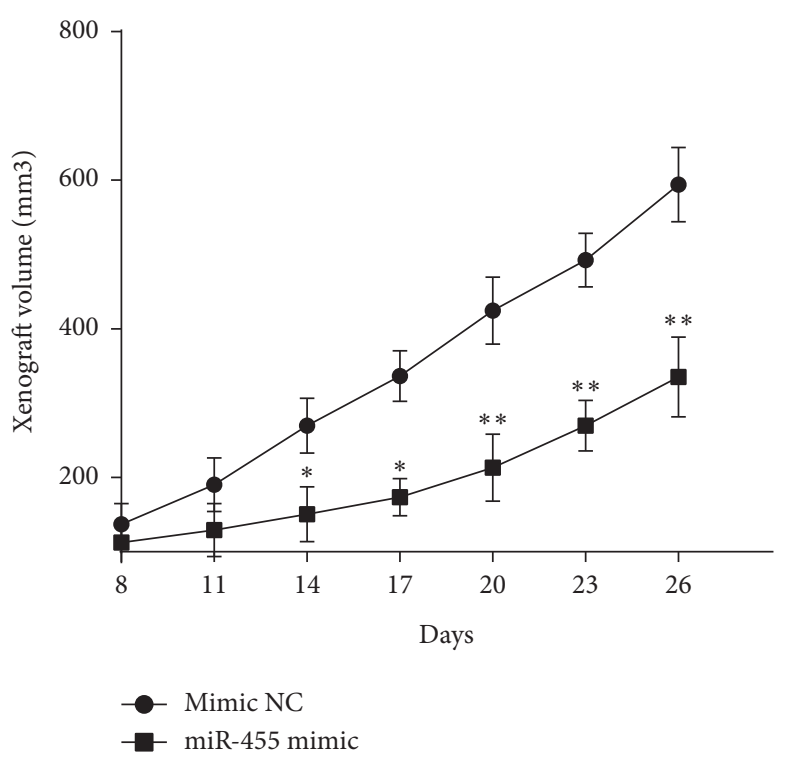

(d)

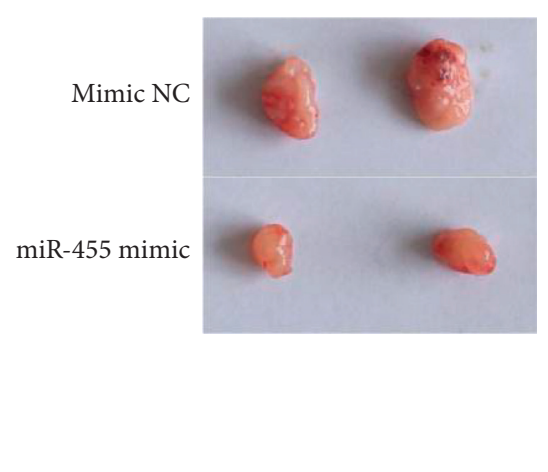

FIGURE 2: miR-455 inhibits cell viability of HCC in vitro and in vivo. (a) miR-455 mimic and miR-455 inhibitor were used to upregulate or downregulate the expression of miR-455 vs mimic NC or inhibitor $\mathrm{NC},{ }^{*} P<0.05,{ }^{* *} P<0.01$. (b) The proliferative ability was decreased after transfection with miR-455 mimic vs mimic NC, ${ }^{*} P<0.05$. (c) miR-455 inhibitor increased proliferative ability in comparison with normal control vs inhibitor NC, ${ }^{*} P<0.05$. (d) miR-455 suppressed the growth of HCC in vivo vs inhibitor NC, ${ }^{*} P<0.05 ;{ }^{* *} P<0.01$. (e) Tumors of miR-455 overexpressed group had a smaller volume than that of control group vs inhibitor $\mathrm{NC},{ }^{*} \mathrm{P}<0.05$.

Transwell assays were performed in miR-455 mimic-transfected Huh7 cells. RAB18 overexpressed plasmid was transfected in miR-455 mimic-transfected cells and the efficiency was calculated by RT-qPCR (Figure 5(c)). CCK8 result revealed that cotransfection with RAB18 overexpressed plasmid and miR-455 mimic increased cell proliferation versus only transfected miR-455 mimic $(P<0.05)$ (Figure 5(d)). Moreover, Transwell assay elucidated that overexpressing RAB18 improved cell invasive ability in miR455 mimic-transfected cells $(P<0.05)$ (Figure 5(e)). All results validated that RAB18 reversed partial functions of miR455 on cell viability and invasion in HCC cells.

\section{Discussion}

Although treatment with HCC has improved, recurrence and metastasis often occur, and the 5-year overall survival rate remains low $[23,24]$. Therefore, it is important to identify growth and metastasis mechanisms and develop biomarkers that improve patient outcomes.

Increasing evidence indicates that miRNAs have a major impact on cancer growth and metastasis and are associated with tumor development and progression $[25,26]$. A previous study showed that miR-455 functioned as a tumor suppressor to inhibit cell proliferation and migration in 


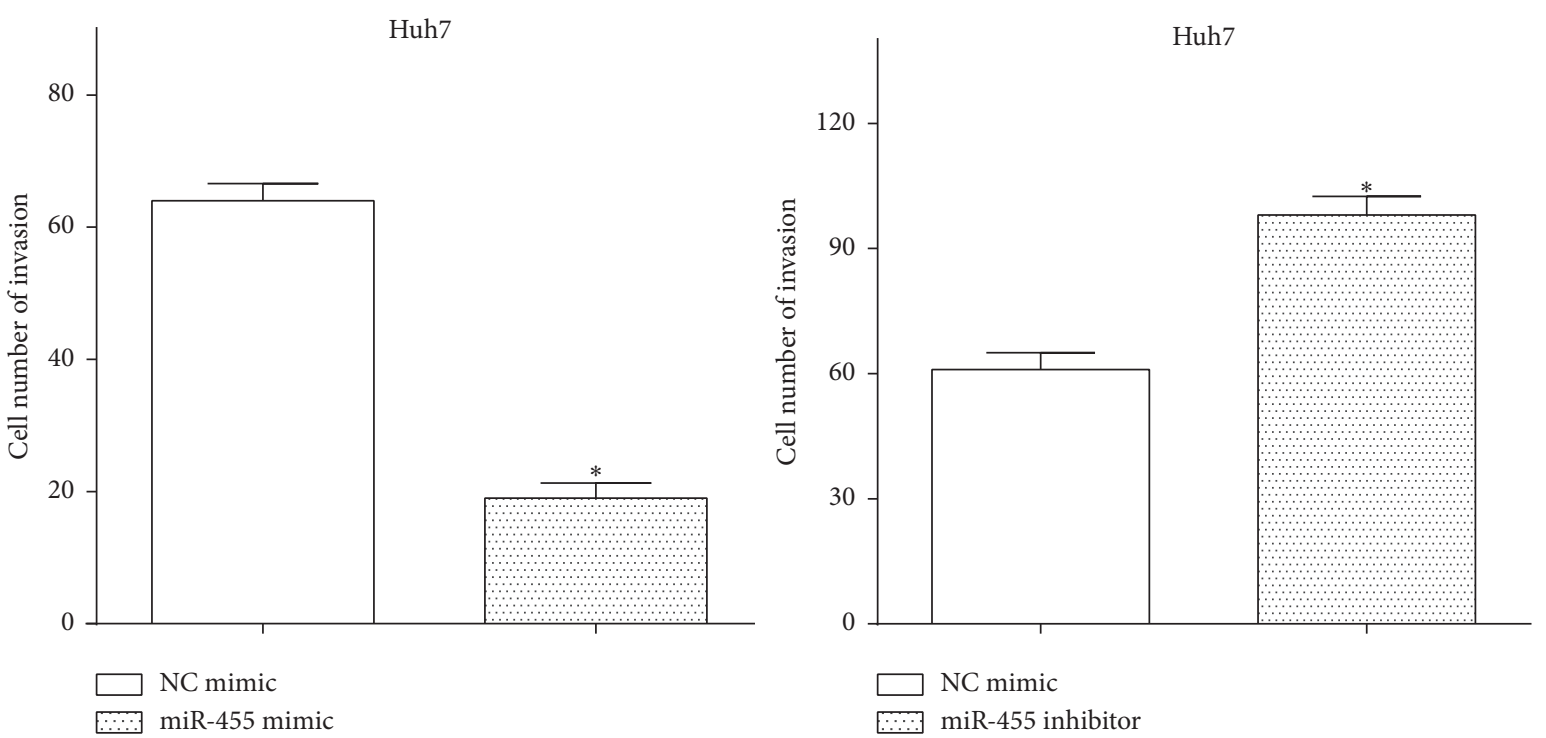

Huh7

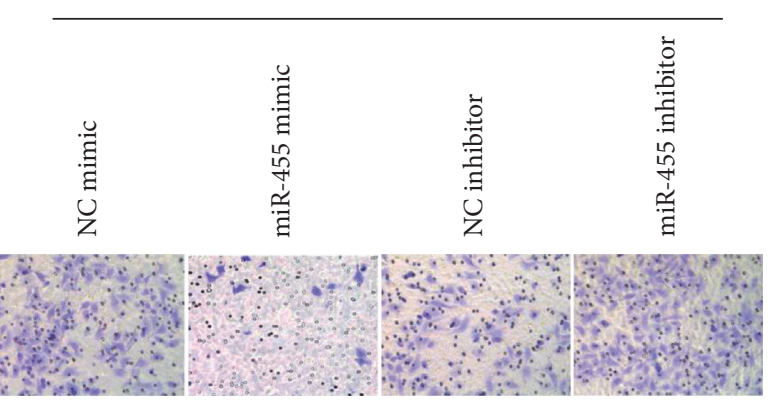

(a)

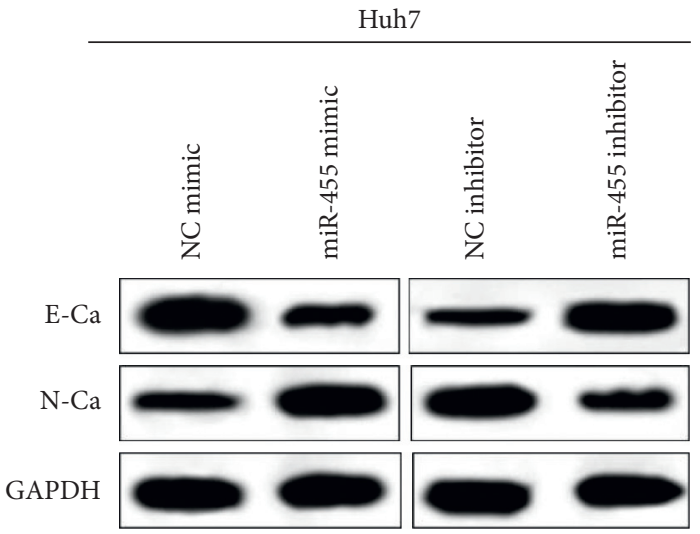

(b)

FIGURE 3: miR-455 impairs cell metastasis of HCC cells. (a) miR-455 mimic reduced the invasive ability, while miR-455 inhibitor improved that in Huh7 cells vs mimic NC, ${ }^{*} P<0.05$. (b) miR-455 inhibited the EMT ability in Huh7 cells.

colorectal cancer [27]. Methods were referred to previous studies [28]. Consistent with the findings in colorectal cancer, we discovered that the expression of miR-455 was low in HCC tissues and cell lines versus the nontumor tissues and normal cells. What is more, our results were consistent with the findings in esophageal squamous cell carcinoma, miR-455 acted as a prognostic marker, and downregulation of miR-455 was connected with worse outcome of HCC patients [29]. miR-455 suppressed cell proliferation of HCC in vitro and in vivo, which was consistent with the findings in breast cancer [30]. What is more, miR-455 impaired cell proliferation and invasion in colorectal cancer [31]. We also found that miR-455 suppressed cell invasive ability in HCC. In addition, miR-455 suppressed the EMT phenomenon of HCC by downregulating N-cadherin expression but upregulating E-cadherin.

RAB18 promoted cell viability, invasion, and migration and impaired cell apoptosis in gastric cancer [32]. In addition, 


\begin{tabular}{|c|c|}
\hline \multicolumn{2}{|c|}{ Site: $96-102$ of RAB18 } \\
\hline $3^{\prime} \mathrm{UTR}$ WT & $5^{\prime}$-...GGGACCUUGCAGUUUGCACAUAA....3' \\
\hline hsa-miR-455 & $3^{\prime}$-...GCUACAUCAGGUUUCCGUGUAU....5' \\
\hline 3'UTR MUT & $5^{\prime}$-...GGGACCUUGCAGUUUCGUGUAUA....3' \\
\hline
\end{tabular}

(a)

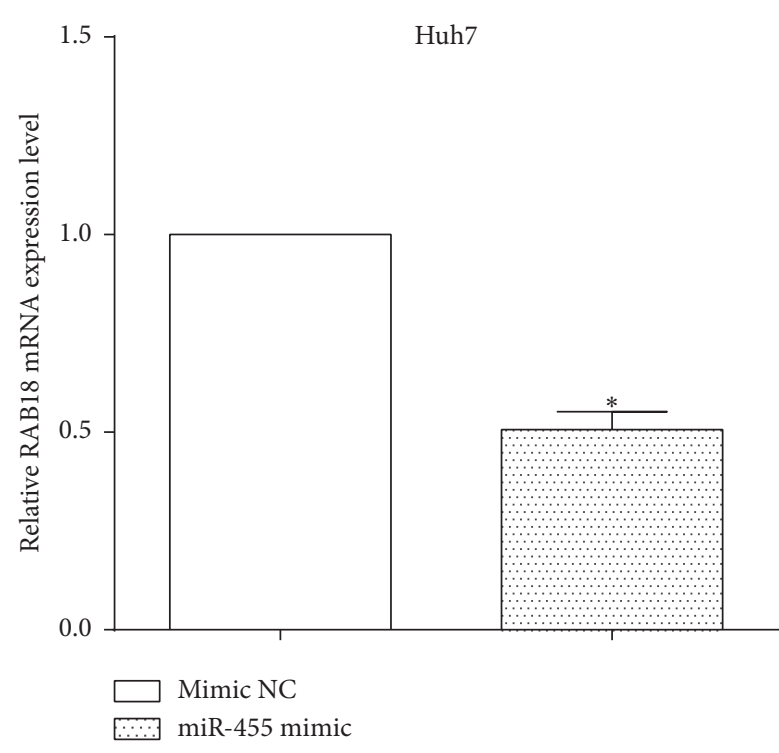

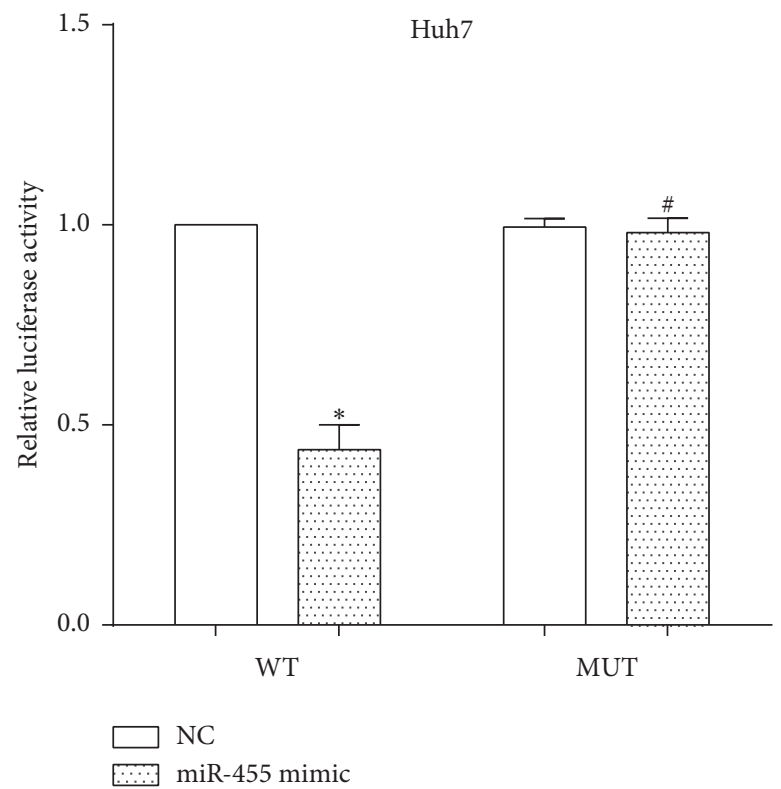

(b)

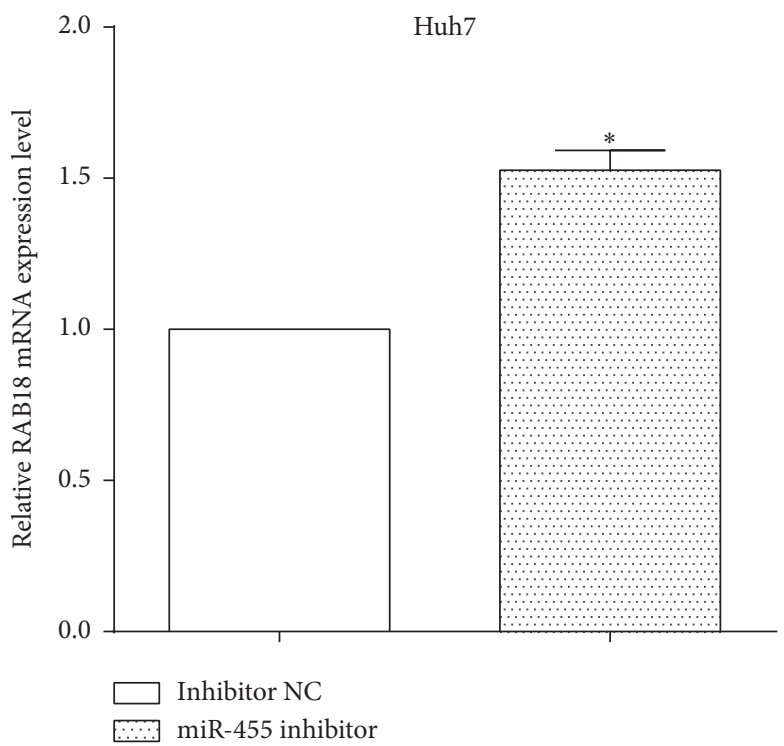

(c)

Figure 4: miR-455 targets RAB18 and regulates its expression. (a) TargetScan predicted that RAB18 was a potential target of miR-455. (b) miR-455 mimic reduced the luciferase ability of wild type mRNA $3^{\prime}$-UTR, whereas it did not alter the mutant $3^{\prime}$-UTR vs WT-NC, ${ }^{*} P<0.05$ and vs MUT-NC, ${ }^{\#} P<0.05$. (c) The expression of RAB18 was decreased by miR-455 mimic, while it was promoted by miR-455 inhibitor in Huh7 cells vs mimic NC, ${ }^{*} P<0.05$ and vs inhibitor NC, ${ }^{*} P<0.05$.

interference of RAB18 suppressed cell viability of non-smallcell lung cancer [33]. In this study, the expression of RAB18 was significantly mediated by miR-455, which was consistent with the findings in gastric cancer where miR-455 targeted
RAB18 through directly binding to the $3^{\prime}$-UTR of RAB18 mRNA [34]. What is more, we discovered that RAB18 was overexpressed in HCC tissues and cell lines. RAB18 reversed partial roles of miR-455 on cell viability and invasion in HCC. 


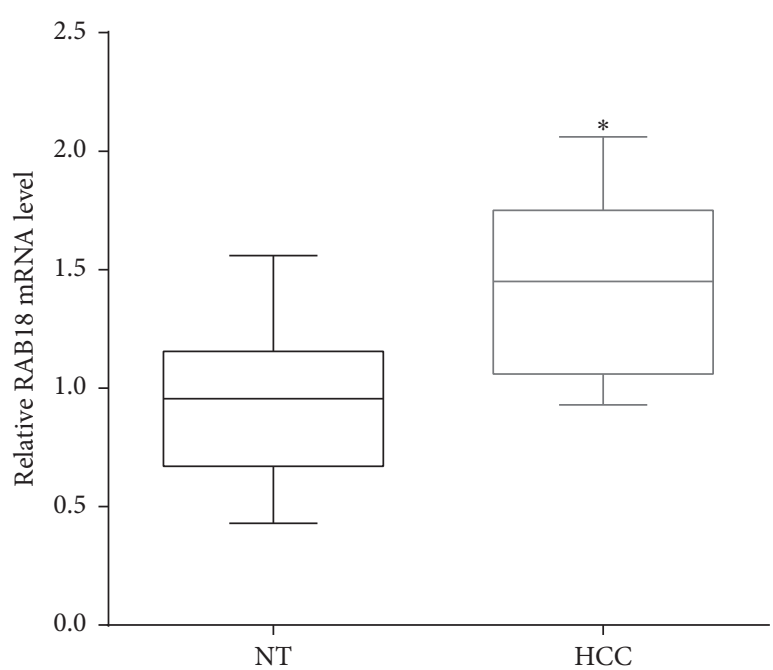

(a)

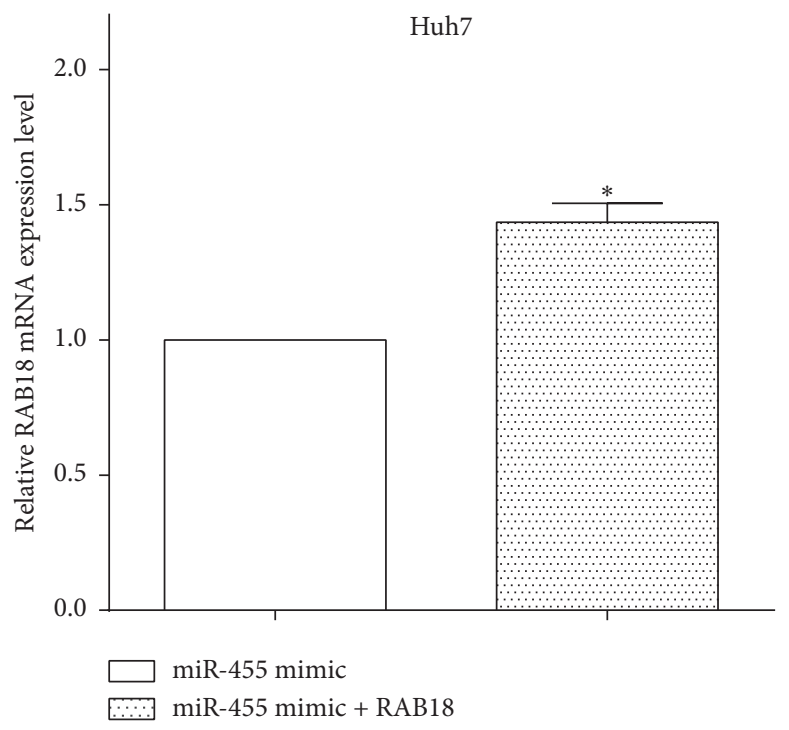

(c)

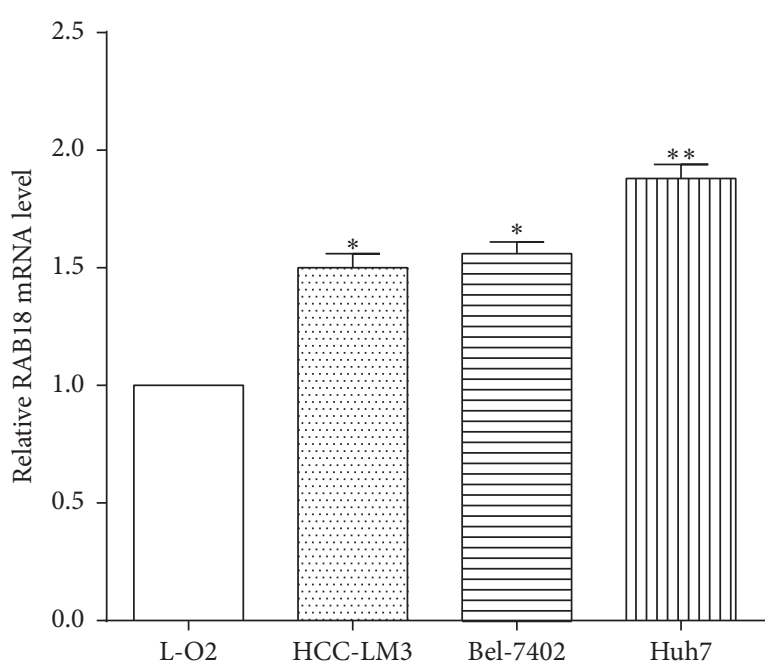

(b)

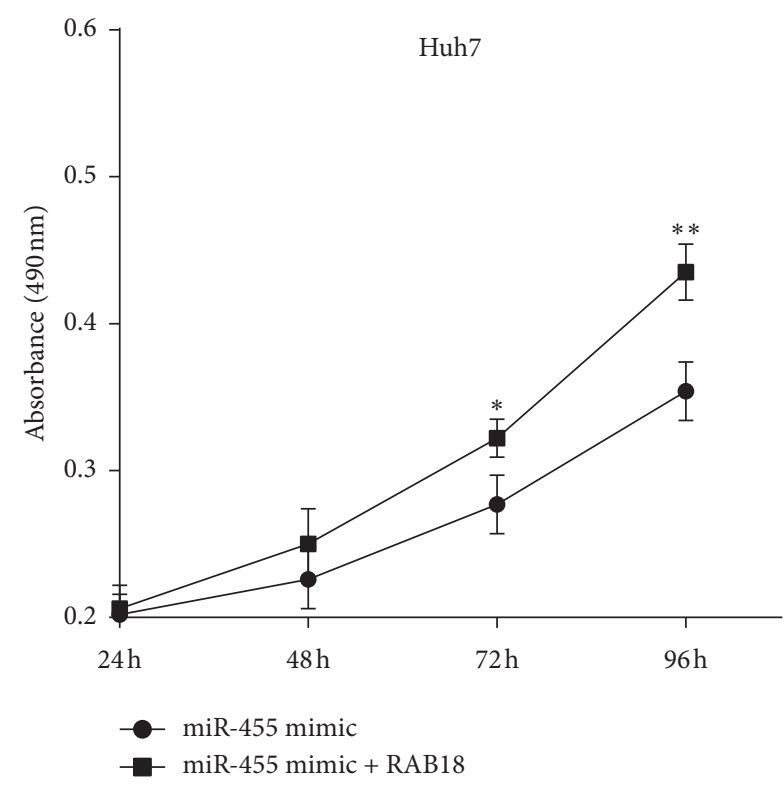

(d)

Figure 5: Continued. 


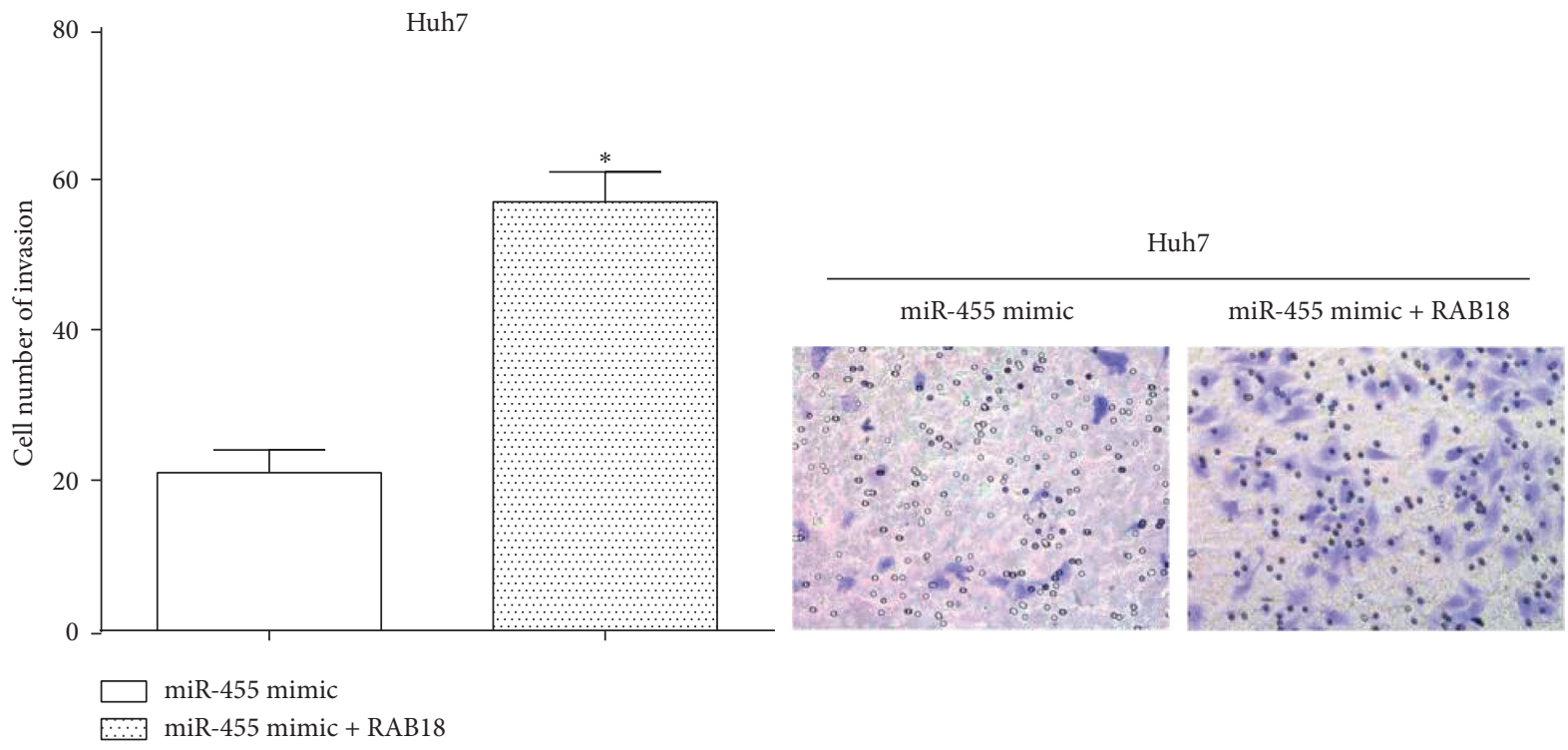

(e)

Figure 5: RAB18 restores partial functions of miR-455 on cell viability and invasion. (a) RAB18 was overexpressed in HCC tissues versus nontumor tissues vs NT, ${ }^{*} P<0.05$. (b) The expression of RAB18 was higher in HCC-LM3, Huh7, and Bel-7402 than in L-O2 cells vs L-O2, ${ }^{*} P<0.05 ;{ }^{* *} P<0.01$. (c) RT-qPCR revealed the transfection efficiency of overexpressing RAB18 in miR-455 mimic-tranfected cells vs miR455 mimic, ${ }^{*} P<0.05$. (d) RAB18 reversed partial functions of miR-455 on cell viability vs miR-455 mimic, ${ }^{*} P<0.05$. (e) Overexpressing RAB18 improved cell invasive ability in miR-455 mimic-transfected cells vs miR-455 mimic, ${ }^{*} P<0.05$.

\section{Conclusion}

The expression of miR-455 was low in HCC tissues and cell lines, and downregulation of miR-455 was connected with worse outcome of HCC patients. miR-455 suppressed cell growth in vitro and in vivo and suppressed the abilities of cell invasion and EMT in HCC. miR-455 regulated cell viability and invasion by directly targeting the 3 '-UTR of RAB18 mRNA. RAB18 reversed partial roles of miR-455 on cell viability and invasion in HCC.

\section{Data Availability}

The data used to support the findings of this study are available from the corresponding author upon request.

\section{Conflicts of Interest}

The authors declare that they have no conflicts of interest.

\section{References}

[1] A. Forner and J. Bruix, "Biomarkers for early diagnosis of hepatocellular carcinoma," The Lancet Oncology, vol. 13, no. 8, pp. 750-751, 2012.

[2] H. C. Spangenberg, R. Thimme, and H. E. Blum, "Targeted therapy for hepatocellular carcinoma," Nature Reviews Gastroenterology and Hepatology, vol. 6, no. 7, pp. 423-432, 2009.

[3] S. Kakar, J. P. Grenert, V. Paradis, N. Pote, S. Jakate, and L. D. Ferrell, "Hepatocellular carcinoma arising in adenoma: similar immunohistochemical and cytogenetic features in adenoma and hepatocellular carcinoma portions of the tumor," Modern Pathology, vol. 27, no. 11, pp. 1499-1509, 2014.
[4] A. P. Venook, C. Papandreou, J. Furuse, and L. Ladrón de Guevara, "The incidence and epidemiology of hepatocellular carcinoma: a global and regional perspective," The Oncologist, vol. 15, no. S4, pp. 5-13, 2010.

[5] L. Liu, L. Chen, X. Wu et al., "Low-dose DNA-demethylating agent enhances the chemosensitivity of cancer cells by targeting cancer stem cells via the upregulation of microRNA497," Journal of Cancer Research and Clinical Oncology, vol. 142, no. 7, pp. 1431-1439, 2016.

[6] S. M. Hartig, M. P. Hamilton, D. A. Bader, and S. E. McGuire, "The miRNA interactome in metabolic homeostasis," Trends in Endocrinology and Metabolism, vol. 26, no. 12, pp. 733-745, 2015.

[7] A. Habieb, M. Matboli, H. El-Tayeb, and F. El-Asmar, "Potential role of IncRNA-TSIX, miR-548-a-3p, and SOGA1 mRNA in the diagnosis of hepatocellular carcinoma," Molecular Biology Reports, vol. 46, pp. 4581-4590, 2019.

[8] M. Chuma, H. Toyoda, J. Matsuzaki et al., "Circulating microRNA-1246 as a possible biomarker for early tumor recurrence of hepatocellular carcinoma," Hepatology Research: The Official Journal of the Japan Society of Hepatology, vol. 49, pp. 810-822, 2019.

[9] J. Pu, J. Wang, Z. Xu et al., "miR-632 functions as oncogene in hepatocellular carcinoma via targeting MYCT1," Human Gene Therapy. Clinical development, vol. 30, pp. 67-73, 2019.

[10] S. Sun, N. Wang, Z. Sun, X. Wang, and H. Cui, "MiR-5692a promotes proliferation and inhibits apoptosis by targeting HOXD8 in hepatocellular carcinoma," Journal of B.U.ON.: Official Journal of the Balkan Union of Oncology, vol. 24, pp. 178-186, 2019.

[11] Y. Zhao, M. Yan, Y. Yun et al., "MicroRNA-455-3p functions as a tumor suppressor by targeting eIF4E in prostate cancer," Oncology Reports, vol. 37, no. 4, pp. 2449-2458, 2017.

[12] T. Ning, Z. Peng, S. Li et al., "miR-455 inhibits cell proliferation and migration via negative regulation of EGFR in human gastric cancer," Oncology Reports, vol. 38, no. 1, pp. 175-182, 2017. 
[13] Q. D. Mao, W. Zhang, K. Zhao et al., "MicroRNA-455 suppresses the oncogenic function of HDAC2 in human colorectal cancer," Brazilian Journal of Medical and Biological Research, vol. 50, Article ID e6103, 2017.

[14] Z. Li, Q. Meng, A. Pan et al., "MicroRNA-455-3p promotes invasion and migration in triple negative breast cancer by targeting tumor suppressor EI24," Oncotarget, vol. 8, no. 12, pp. 19455-19466, 2017.

[15] W. J. Chia and B. L. Tang, "Emerging roles for Rab family GTPases in human cancer," Biochimica et Biophysica Acta (BBA)-Reviews on Cancer, vol. 1795, no. 2, pp. 110-116, 2009.

[16] M. Huang and Y. Wang, "Targeted quantitative proteomic approach for probing altered protein expression of small GTPases associated with colorectal cancer metastasis," Analytical Chemistry, vol. 91, pp. 6233-6241, 2019.

[17] V. Casado-Medrano, L. Barrio-Real, A. Wang, M. Cooke, C. Lopez-Haber, and M. G. Kazanietz, "Distinctive requirement of PKC $\varepsilon$ in the control of Rho GTPases in epithelial and mesenchymally transformed lung cancer cells," Oncogene, vol. 38, pp. 5396-5412, 2019.

[18] P. Seshacharyulu, S. Rachagani, S. Muniyan et al., "FDPS cooperates with PTEN loss to promote prostate cancer progression through modulation of small GTPases/AKT axis," Oncogene, vol. 38, pp. 5265-5280, 2019.

[19] D. Xu, Y. Li, L. Wu et al., "Rab18 promotes lipid droplet (LD) growth by tethering the ER to LDs through SNARE and NRZ interactions," Journal of Cell Biology, vol. 217, no. 3, pp. 975-995, 2018.

[20] S. Salloum, H. Wang, C. Ferguson, R. G. Parton, and A. W. Tai, "Rab18 binds to hepatitis C virus NS5A and promotes interaction between sites of viral replication and lipid droplets," PLoS Pathogens, vol. 9, Article ID e1003513, 2013.

[21] R. Vazquez-Martinez, A. J. Martinez-Fuentes, M. R. Pulido et al., "Rab18 is reduced in pituitary tumors causing acromegaly and its overexpression reverts growth hormone hypersecretion," The Journal of Clinical Endocrinology \& Metabolism, vol. 93, no. 6, pp. 2269-2276, 2008.

[22] M. R. Pulido, A. Diaz-Ruiz, Y. Jimenez-Gomez et al., "Rab18 dynamics in adipocytes in relation to lipogenesis, lipolysis and obesity," PLoS One, vol. 6, Article ID e22931, 2011.

[23] J. Bruix, G. J. Gores, and V. Mazzaferro, "Hepatocellular carcinoma: clinical frontiers and perspectives," Gut, vol. 63, no. 5, pp. 844-855, 2014.

[24] G. Ferrin, M. de la Mata, J. L. Montero-Álvarez, P. AguilarMelero, and M. Rodríguez-Perálvarez, "Biomarkers for hepatocellular carcinoma: diagnostic and therapeutic utility," Hepatic Medicine: Evidence and Research, vol. 7, pp. 1-10, 2015.

[25] D. P. Bartel, "MicroRNAs: target recognition and regulatory functions," Cell, vol. 136, no. 2, pp. 215-233, 2009.

[26] G. A. Calin and C. M. Croce, "MicroRNA signatures in human cancers," Nature Reviews Cancer, vol. 6, no. 11, pp. 857-866, 2006.

[27] H. Yunqi, Y. Fangrui, Y. Yongyan et al., "miR-455 functions as a tumor suppressor through targeting GATA6 in colorectal cancer," Oncology Research Featuring Preclinical and Clinical Cancer Therapeutics, vol. 27, no. 3, pp. 311-316, 2019.

[28] Y. Jiao, G. Zhu, J. Yu et al., "miR-1271 inhibits growth, invasion and epithelial-mesenchymal transition by targeting ZEB1 in ovarian cancer cells," OncoTargets and Therapy, vol. 12, pp. 6973-6980, 2019.
[29] H. Yang, Y. N. Wei, J. Zhou, T. T. Hao, and X. L. Liu, "MiR455-3p acts as a prognostic marker and inhibits the proliferation and invasion of esophageal squamous cell carcinoma by targeting FAM83F," European Review for Medical and Pharmacological Sciences, vol. 21, pp. 3200-3206, 2017.

[30] B. Wang, A. Zou, L. Ma et al., "miR-455 inhibits breast cancer cell proliferation through targeting CDK14," European Journal of Pharmacology, vol. 807, pp. 138-143, 2017.

[31] J. Chai, S. Wang, D. Han, W. Dong, C. Xie, and H. Guo, "MicroRNA-455 inhibits proliferation and invasion of colorectal cancer by targeting RAF proto-oncogene serine/threonine-protein kinase," Tumor Biology, vol. 36, no. 2, pp. 1313-1321, 2015.

[32] B. Wu, R. Qi, X. Liu, L. Qian, and Z. Wu, "Rab18 overexpression promotes proliferation and chemoresistance through regulation of mitochondrial function in human gastric cancer," OncoTargets and Therapy, vol. 11, pp. 78057820, 2018.

[33] K. Zhong, K. Chen, L. Han, and B. Li, "MicroRNA-30b/c inhibits non-small cell lung cancer cell proliferation by targeting Rab18," BMC Cancer, vol. 14, p. 703, 2014.

[34] J. Liu, J. Zhang, Y. Li, L. Wang, B. Sui, and D. Dai, “MiR-455$5 \mathrm{p}$ acts as a novel tumor suppressor in gastric cancer by downregulating RAB18," Gene, vol. 592, no. 2, pp. 308-315, 2016. 